\title{
Hilar portal vein wedge resection and patch venoplasty in patients undergoing bile duct resection for hepatobiliary malignancy: A report of two cases
}

\author{
Sung-Min Kim and Shin Hwang
}

\author{
Department of Surgery, Asan Medical Center, University of Ulsan College of Medicine, Seoul, Korea
}

\begin{abstract}
Margin-free resection is one of the most important factors for favorable prognosis in patients undergoing resection for hepatobiliary malignancies. Herein, we present two cases of hepatobiliary malignancies in patients who underwent bile duct resection combined with hilar portal vein (PV) resection and vein allograft patch plasty. The first case was a 51-year-old female patient with gallbladder cancer, in whom we performed extended cholecystectomy, bile duct resection and extensive lymph node dissection. The tumor-invaded PV wall was meticulously excised and the defect was repaired with a cryopreserved iliac vein allograft patch. The extent of the tumor was pT4N2M0 (stage IVB), thus concurrent chemoradiation therapy and adjuvant chemotherapy were performed. This patient is currently alive for 7 years after surgery without any evidence of tumor recurrence. The second case was a 79-year-old male patient with perihilar cholangiocarcinoma of type I and gallbladder cancer, in whom extended bile duct resection and extensive lymph node dissection were performed. The extent of the bile duct tumor was pT4N1M0 (stage IVA) and that of gallbladder tumor was PT2NOMO (stage II). No additional treatment was provided because of old age and poor general condition. This patient passed away 11 months after surgery due to rapid progression of tumor recurrence. In conclusion, hilar PV wedge resection and roofing patch venoplasty is a useful option to facilitate complete tumor resection in patients undergoing bile duct resection for hepatobiliary malignancy. (Ann Hepatobiliary Pancreat Surg 2021;25:132-138)
\end{abstract}

Key Words: Bile duct resection; Portal vein; Perihilar cholangiocarcinoma; Gallbladder cancer; Vascular reconstruction

\section{INTRODUCTION}

Hepatobiliary malignancies located at the hepatic hilum often invade the portal vein (PV) because the perihilar bile duct lies in close proximity to the PV. Margin-free resection is one of the most important factors for favorable prognosis in patients undergoing resection for hepatobiliary malignancies. ${ }^{1-3} \mathrm{PV}$ resection combined with hepatectomy has been frequently performed for patients with perihilar cholangiocarcinoma in many centers worldwide. On the contrary, PV resection combined with only bile duct resection (BDR) has been rarely performed because of the low incidence of the indicated cases. When the local direct invasion of the PV is identified during BDR for hepatobiliary malignancies, the surgical techniques for PV resection can be different depending on the invasion site. If the main PV is involved, segmental resection and end-to-end anastomosis or wedge resection with primary repair can be performed because the main PV can obtain some redundancy through extensive PV mobilization. However, if the PV bifurcation is invaded, wedge resection of the involved PV portion and roofing patch venoplaty are the preferred procedure. We herein present two cases of hepatobiliary malignancies in patients who underwent BDR combined with hilar PV resection and vein allograft patch plasty.

\section{CASE}

\section{Case 1}

A 51-year-old female patient was referred to our institution under the diagnosis of gallbladder cancer.

Received: October 25, 2020; Revised: November 1, 2020; Accepted: November 3, 2020

Corresponding author: Shin Hwang

Department of Surgery, Asan Medical Center, University of Ulsan College of Medicine, 88 Olympic-ro 43-gil, Songpa-gu, Seoul 05505, Korea Tel: +82-2-3010-3930, Fax: +82-2-3010-6701, E-mail: shwang@amc.seoul.kr

Copyright (C) 2021 by The Korean Association of Hepato-Biliary-Pancreatic Surgery

This is an Open Access article distributed under the terms of the Creative Commons Attribution Non-Commercial License (http://creativecommons.org/ licenses/by-nc/4.0) which permits unrestricted non-commercial use, distribution, and reproduction in any medium, provided the original work is properly cited. Annals of Hepato-Biliary-Pancreatic Surgery • pISSN: 2508-5778 - eISSN: 2508-5859 
Computed tomography (CT) and magnetic resonance imaging (MRI) showed advanced gallbladder cancer with direct hepatic invasion, multiple hepatoduodenal ligament lymph node (LN) metastases, invasion of the main PV and right posterior PV by the metastatic LNs, and common hepatic duct invasion due to metastatic LNs. Fluorodeoxyglucose-positron emission tomography (FDG-PET) scan also showed gallbladder cancer with multiple metastatic
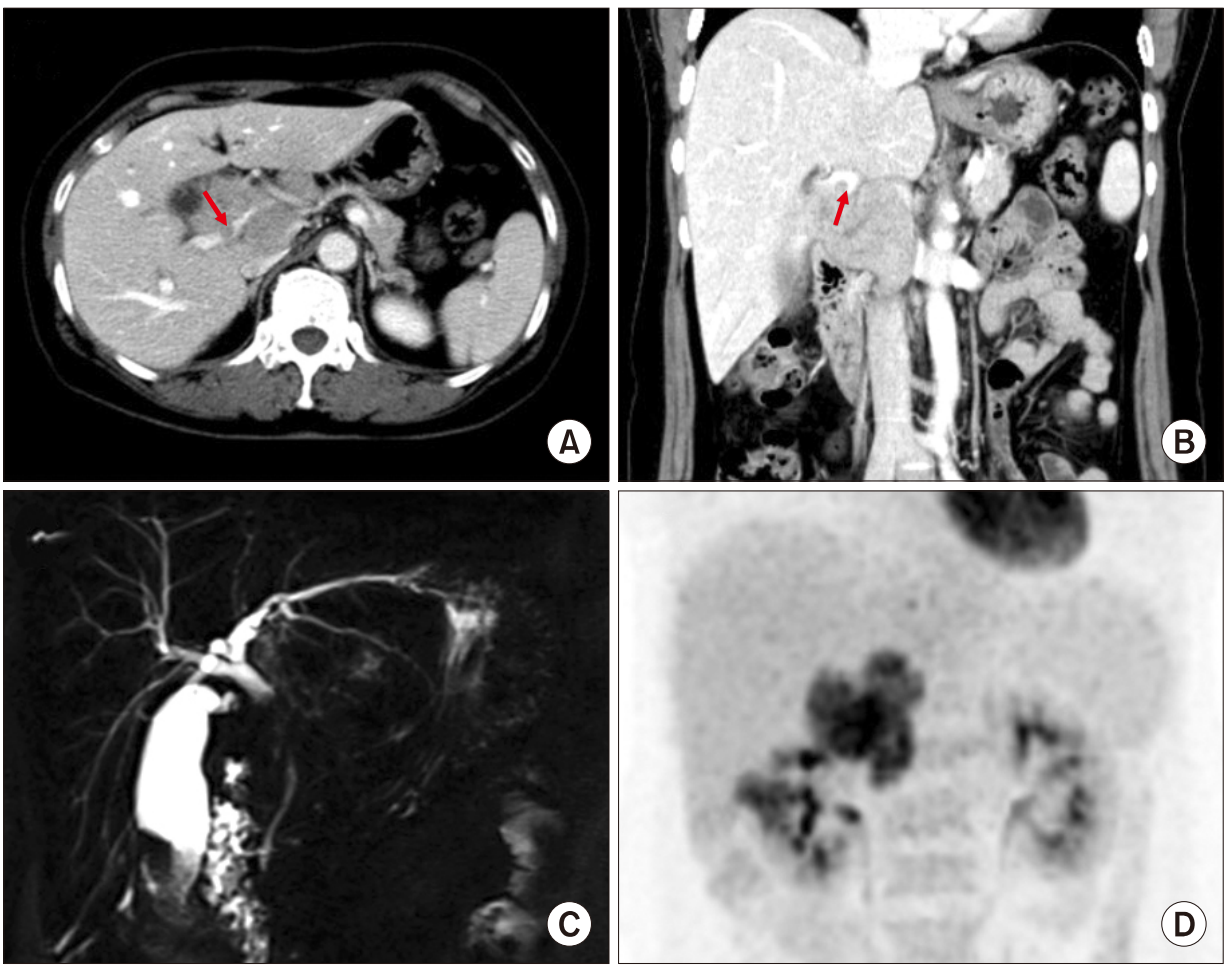

(D)
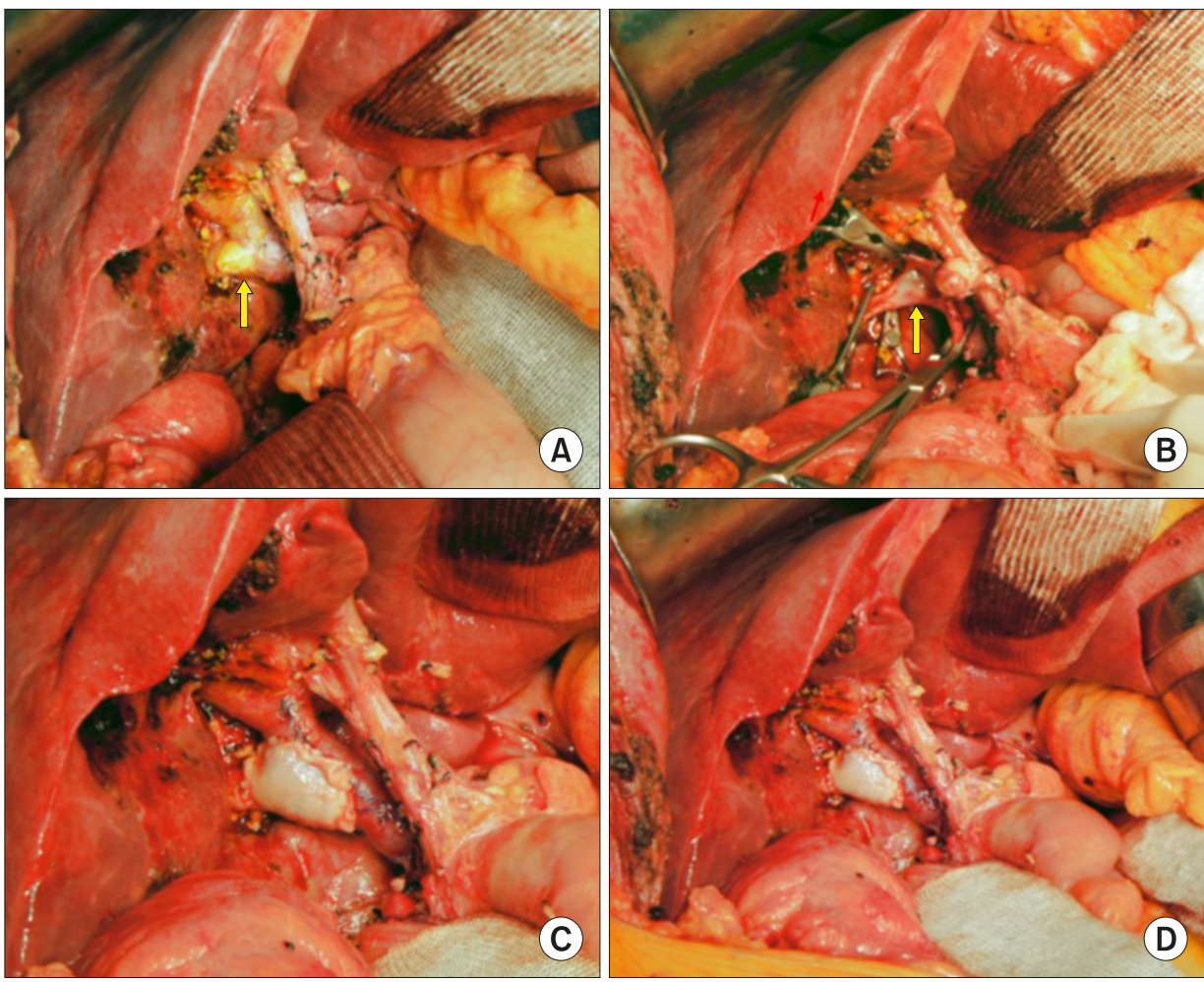

Fig. 1. Preoperative radiologic findings of Case No. 1. (A, B) Computed tomography scan shows advanced gallbladder cancer with multiple hepatoduodenal ligament lymph node metastases and invasion of the right posterior portal vein (arrows). (C) Magnetic resonance cholangiopancreatography shows common hepatic duct invasion by the metastatic lymph nodes. (D) Fluorodeoxyglucosepositron emission tomography scan shows gallbladder cancer with multiple lymph node metastasis.

Fig. 2. Intraoperative photographs of wedge resection of the hilar portal vein (PV) invasion and roof patch venoplasty in Case No. 1. (A) The tumor invaded the branching portion of the right posterior section PV (arrow). (B) The invaded PV wall was excised (arrow). (C, D) The defect at the PV wall was repaired with a cryopreserved iliac vein allograft patch. 
LNs in hepatoduodenal ligament and portocaval area (Fig. 1).

The tumor appeared to be resectable despite extensive lymphadenopathy. Accordingly, we performed extended cholecystectomy, bile duct resection and extensive LN dissection. After dissection of the hepatoduodenal ligament, invasion of the main PV and right posterior PV was identified. Cholecystectomy and bile duct resection were performed and regional LNs were extensively resected. After isolation of the main PV and hilar PV branches, the invaded PV wall was meticulously excised. The defect in the PV wall was repaired with a cryopreserved iliac vein allograft patch (Fig. 2). As a last step of resection, the liver bed of the gallbladder fossa was excised for complete tumor removal. Single Roux-en-Y hepaticojejunostomy was performed for biliary reconstruction.

The pathology report revealed that the gallbladder cancer was an adenosquamous carcinoma measuring $2.5 \times 2 \times 1.5$ $\mathrm{cm}$ in size, located at the fundus of the gallbladder (Fig. $3)$. The depth of tumor invasion was extension to the perimuscular connective tissue with involvement of the cystic duct. The resected PV fragment showed direct tumor invasion. Lymphovascular invasion and perineural invasion were present. All 5 resected LNs were metastatic. The extent of the tumor was pT4N2M0, thus regarded as stage IVB.

This patient recovered uneventfully from the surgery.
The PV resection site appeared to be slightly stenotic on follow-up CT scans, but the portal perfusion to the right posterior section was not disturbed (Fig. 4).

After surgery, concurrent chemoradiation therapy with capecitabine was administered for 6 weeks followed by adjuvant chemotherapy of 6 cycles with Uracil and Tegafur/ Leucovorin (UFT/LV). This patient is currently alive for 7 years after surgery without any evidence of tumor recurrence.

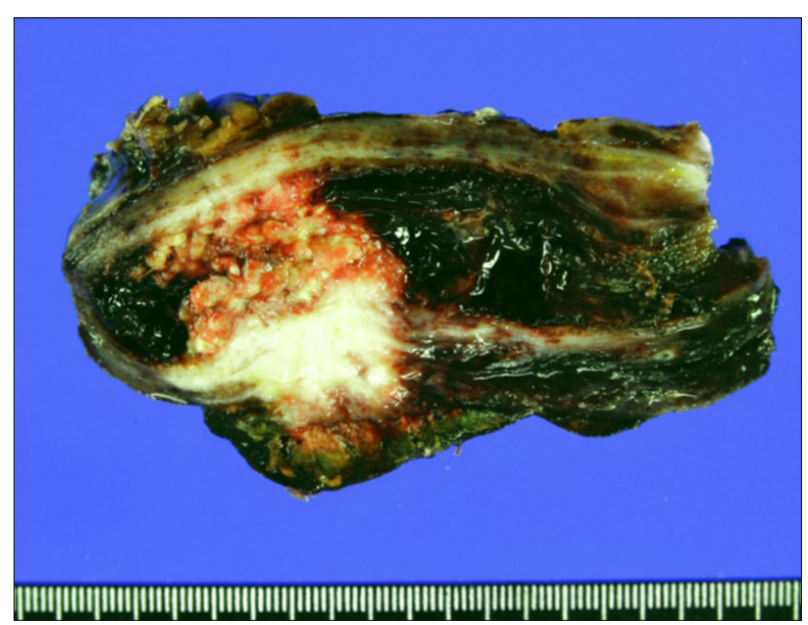

Fig. 3. Gross photographs of Case No. 1 specimen after extended cholecystectomy and bile duct resection showing adenosquamous carcinoma of the gallbladder.
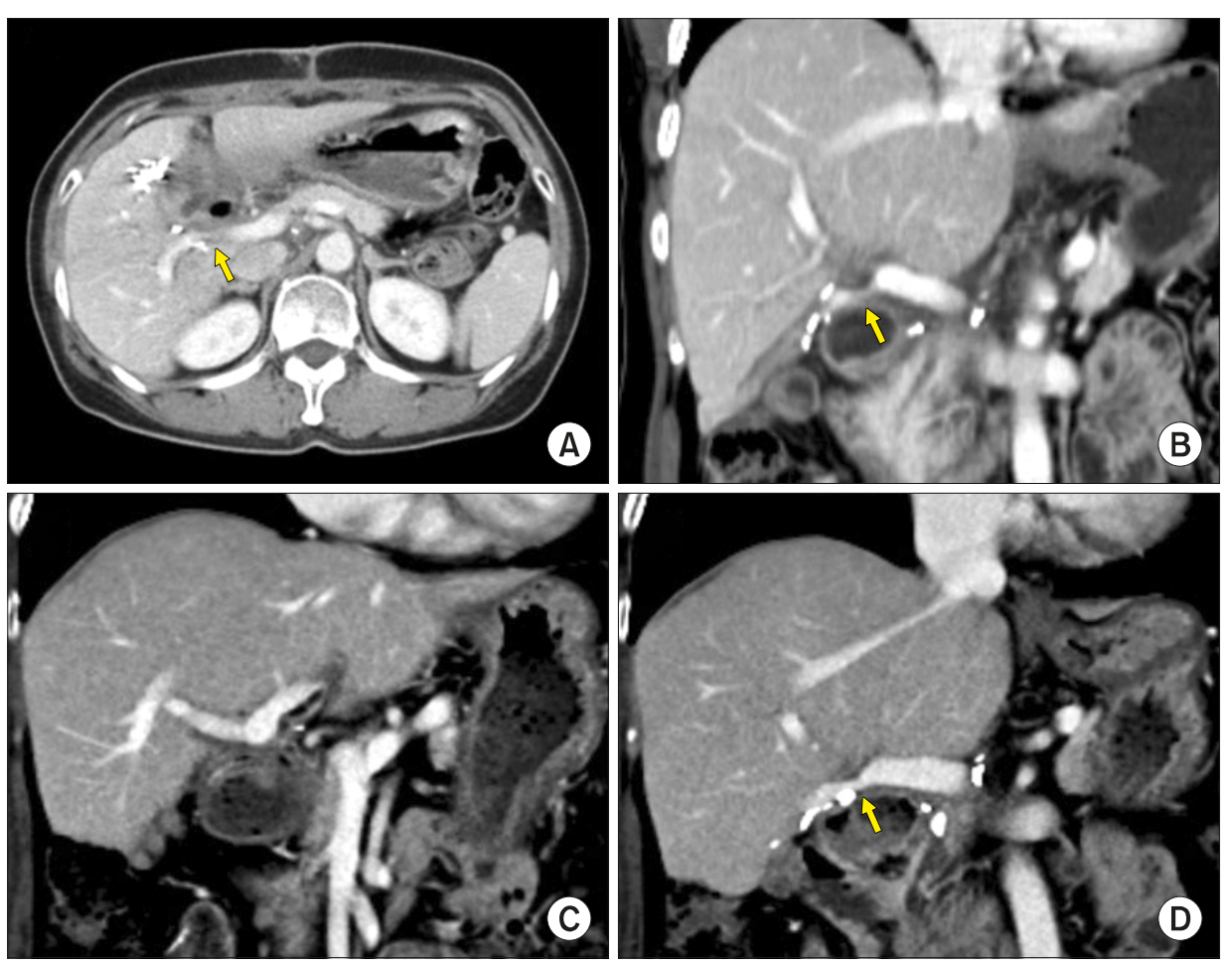

Fig. 4. Follow-up computed tomography scans of Case No. 1 taken at 2 weeks $(\mathrm{A}, \mathrm{B})$ and 6 years $(C, D)$ after the operation. The arrows indicate stenosis at the site of portal vein patch venoplasty. 

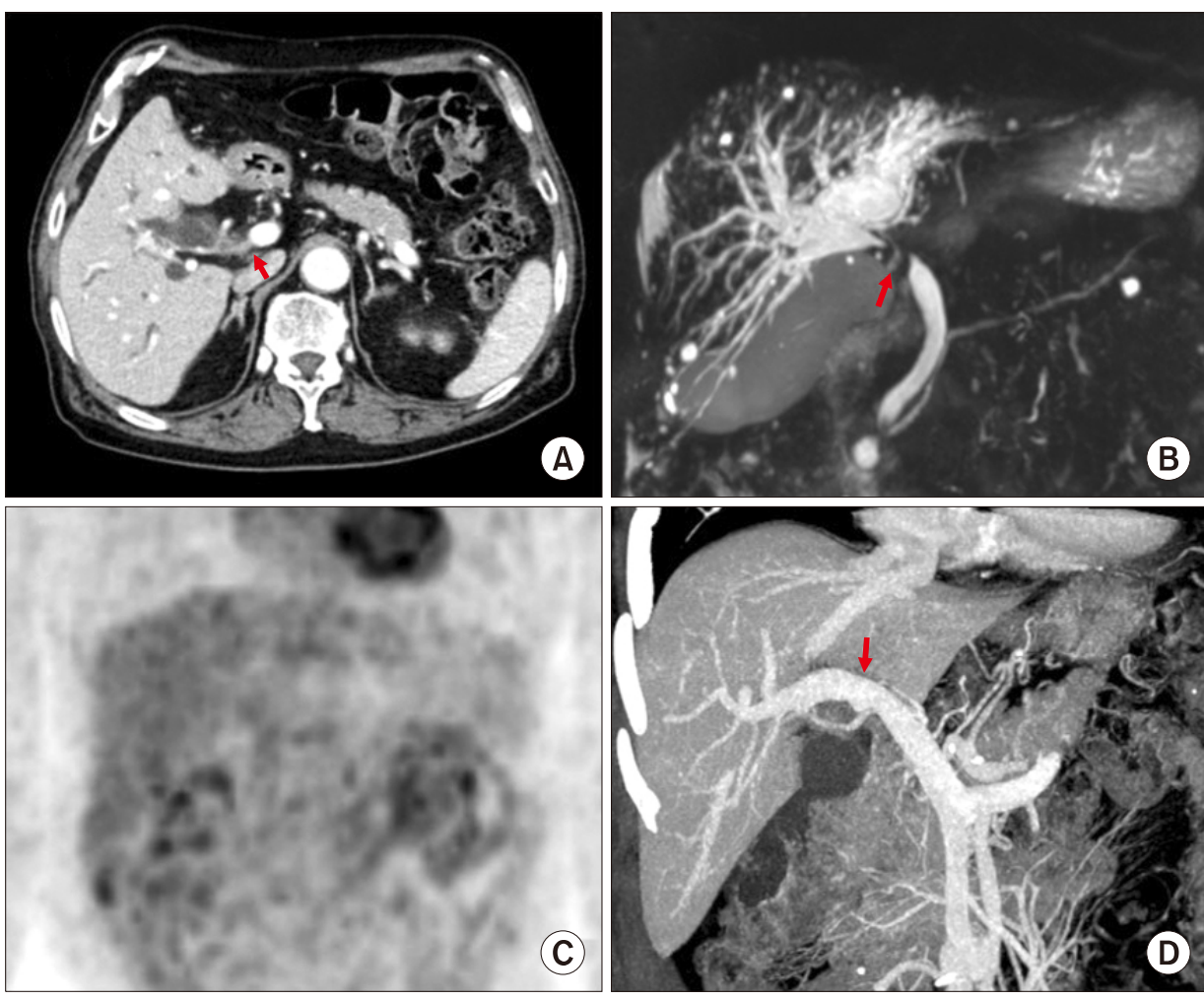

Fig. 5. Preoperative radiologic findings of Case No. 2. (A) Computed tomography scan shows perihilar cholangiocarcinoma of type I (arrow). (B) Magnetic resonance cholangiopancreatography shows the involvement of the hilar bile duct. (C) Fluorodeoxyglucose-positron emission tomography scan shows extrahepatic bile duct cancer. (D) Reconstructed compute tomography image shows the suspected site of portal vein invasion (arrow).

\section{Case 2}

A 79-year-old male patient was referred to our institution under the diagnosis of perihilar bile duct cancer. CT, MRI and FDG-PET showed perihilar cholangiocarcinoma of Bismuth-Corlette type I with suspected PV invasion (Fig. 5). Endoscopic nasobiliary drainage was performed to control obstructive jaundice.

The tumor appeared to be resectable, thus we performed extended bile duct resection after consideration of old age and relatively poor general condition. After dissection of the hepatoduodenal ligament, invasion of the main PV and right PV branch was identified. Cholecystectomy and extended bile duct resection were performed and regional LNs were extensively resected. After isolation of the main PV and hilar PV branches, the invaded PV wall was meticulously excised and the PV defect was repaired with a cryopreserved iliac vein allograft patch (Fig. 6). Clustered hepaticojenunostomy was performed for reconstruction of the multiple hepatic duct openings.

The pathology report revealed double primary tumors at the hilar bile duct and gallbladder. The perihilar bile duct cancer was identified as a cholangiocarcinoma measuring $1.1 \times 0.9 \times 0.7 \mathrm{~cm}$ in size (Fig. 7). The depth of tumor invasion was extension beyond the bile duct wall and the
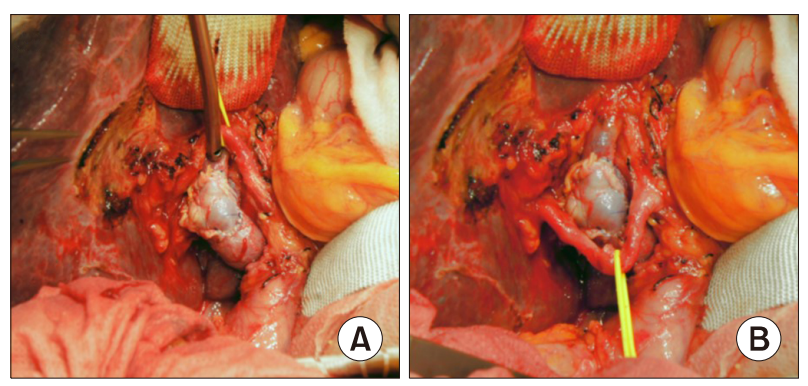

Fig. 6. Intraoperative photographs of the wedge resection of the hilar portal vein $(\mathrm{PV})$ invasion and roof patch venoplasty in Case No. 2. (A) The invaded PV confluence portion is partially excised and repaired with a cryopreserved iliac vein allograft patch. (B) The redundant PV patch is fully expanded.

radial periductal soft tissue resection margins were involved. Lymphovascular invasion and perineural invasion were present. Periarterial neural plexus around the right hepatic artery was tumor-positive. One of the 11 resected LNs were metastatic. The proximal and distal bile duct resection margins were tumor-negative. The extent of the bile duct tumor was pT4N1M0, thus regarded as stage IVA. In addition, the gallbladder mass was moderately differentiated adenocarcinoma at the fundus and body of the gallbladder measuring $4.4 \times 3.0 \times 0.3 \mathrm{~cm}$ in size. The depth of tumor invasion was extension to perimuscular connective tissue 
and perineural invasion were present with absence of lymphovascular invasion. The extent of the gallbladder tumor was pT2N0M0, thus regarded as stage II.

This patient recovered uneventfully from the surgery. Because of the old of the patient and poor general condition, concurrent chemoradiation therapy or adjuvant chemotherapy was not performed. The PV resection site appeared to be slightly stenotic on follow-up CT scans, but PV flow was not disturbed (Fig. 8A). At 6 months after surgery, local recurrence at the hepatic hilum and porto-

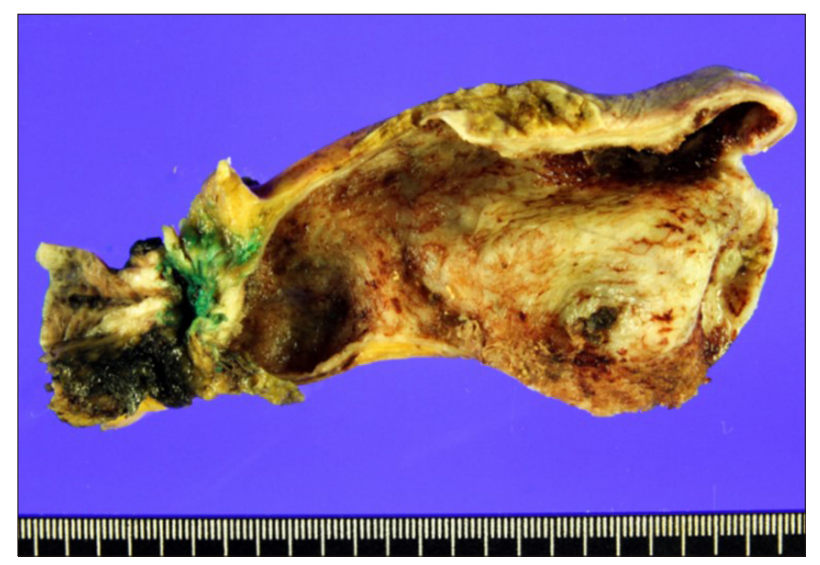

Fig. 7. Gross photographs of Case No. 2 specimen after bile duct resection showing double primary tumors at the hilar bile duct and gallbladder. caval area occurred. As the PV resection site was further stenotic, percutaneous PV stenting was performed (Fig. $8 \mathrm{~B}, \mathrm{C})$. At 8 months after surgery, cholangitis with obstructive jaundice occurred, thus percutaneous biliary wall stenting was performed (Fig. 8D). Because of the rapid progression of tumor recurrence, this patient passed away 11 months after surgery.

\section{DISCUSSION}

Concurrent PV resection during hepatectomy for perihilar cholangiocarcinoma has been considered as a safe procedure ensuring surgical curability. A meta-analysis involving 13 studies including 1921 perihilar cholangiocarcinoma cases revealed that combined PV resection is safe and feasible for the treatment of perihilar cholangiocarcinoma when the PV is grossly involved. In the case of advanced perihilar cholangiocarcinoma, when the portal vein is grossly involved, surgical resection including PV resection can benefit the overall survival in certain patients. ${ }^{1}$ In another meta-analysis involving 11 studies including 371 patients who received PV resection and 1029 who did not, combined PV resection was not significantly associated with higher postoperative mortality. No strong evidence could suggest that combined PV resection leads to
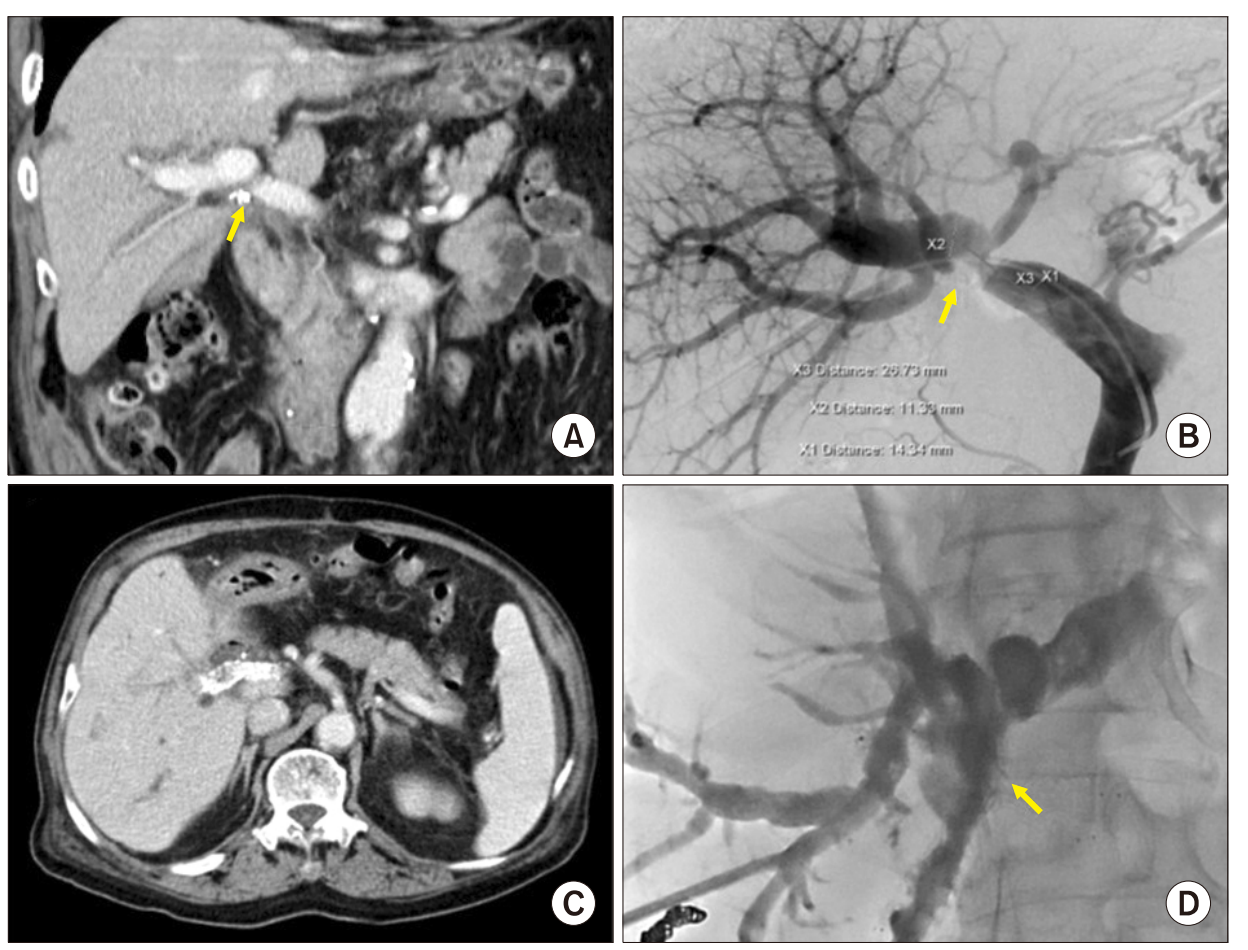

Fig. 8. Follow-up imaging studies of Case No. 2. (A) Computed tomography taken at 4 months shows slight stenosis of the portal vein (PV). (B) At 6 months, the $\mathrm{PV}$ resection site is noticeably stenotic, thus percutaneous PV stenting is performed. (C) Computed tomography scan taken at 7 months shows the expansion of the PV stent with focal stenosis. (D) At 8 months, percutaneous biliary stenting is performed to manage tumor recurrence at the hepaticojejunostomy site. 
more morbidity or mortality in patients with perihilar cholangiocarcinoma when the PV is grossly involved. In addition, combined PV resection is considered oncologically valuable because R0 resection and 5-year survival did not differ significantly between the two cohorts, even though the fact that the PV resection cohort consisted of patients with more advanced perihilar cholangiocarcinoma. ${ }^{2}$ These analyses support that direct PV invasion is a locally controllable extent of cholangiocarcinoma. Thus, it is highly recommended to perform $\mathrm{PV}$ resection if it can lead to margin-free resection.

When performing hemihepatectomy for hepatobiliary malignancies, combined PV resection can be done employing various techniques including wedge or segmental resection combined with primary repair, patch venoplasty and vessel interposition. The extent of PV resection and reconstruction techniques are variable depending on the site and extent of the PV invasion. Meanwhile, when major hepatectomy is not performed, segmental resection of the main or hemihepatic PV branch is technically demanding because the handling of the PV for vascular anastomosis is very difficult. Consequently, the preferred procedure is wedge resection of the involved PV portion and roofing patch venoplaty, as presented in this study.

Unlike primary repair or end-to-end anastomosis, roofing patch venoplaty essentially requires a vessel patch. A cold-stored fresh thick-walled vein allograft, such as external iliac vein, is the best material for patch venoplasty; however, such an allograft vein is usually not available in most of the centers. The next suitable patch material is cryopreserved vein allograft, which is stored at the tissue bank. In both of our cases, we used cryopreserved external iliac vein allograft patches, which were preserved at the tissue bank of our institution. Under the conditions of unavailability of such vein allografts, an autologous vein patch can be considered. The patient's own greater saphenous vein can be converted to a sizable paneled vein patch through a longitudinal incision of the vein and suturing to double the patch size. ${ }^{4,5}$ We do not recommend to the use of any prosthetic vascular graft for patch venoplasty because it is much more thrombogenic than the autologous or homologous vein grafts. The PV is a mediumvelocity high-flow vessel; thus, thus anticoagulation is usually not necessary if the luminal diameter of the PV is maintained. On the contrary, the development of focal steno- sis can lead to a high risk of PV thrombosis, thus hemodynamics-compliant design for PV resection and vascular reconstruction is essential.

Regarding roofing patch venoplasty, it is important to make the PV wall defect as small as possible as well as to make the size of the vein patch either twice or three times larger than the defect size at the PV wall. The patch should be large enough to make the patch roof redundant. Even if the patch roof appears to be noticeably redundant at the time of operation, it is vulnerable to shrinkage due to compression of the jejunal loop and through degenerative changes of the patch per se. Such a shrunken configuration is well demonstrated in in both of our cases. Case No. 1 showed mildly stenotic configuration in follow-up CT scans, but PV flow was well maintained because the cause of PV stenosis was probably extrinsic compression by the jejunal bowel loop crossing the PV. On the contrary, Case No. 2 showed concentric PV stenosis, which was probably associated with degeneration of the patch vein allograft along with extrinsic compression by local tumor recurrence. Further PV stenosis which could induce hepatic dysfunction and portal hypertension was anticipated, thus PV stenting was performed preemptively. ${ }^{6-8}$

Availability of vein allograft facilitates combined vascular resection and reconstruction during aggressive surgery for hepatobiliary malignancies, vascular surgery and living donor liver transplantation. ${ }^{9-11}$ Currently, cryopreserved femoral vein and artery homografts are commercially available through the Korea Public Tissue Bank. All human tissues were donated and stored at the tissue bank after informed consent from the donors' family members. All procedures for vascular tissue procurement and processing were complied with Korean legislation and conformed to the ethical and safety concerns for therapeutic use. $^{12}$

Cluster hepaticojejunostomy technique consisted of applying multiple internal biliary stents and a single wide porto-enterostomy to the surrounding connective tissues, which can be applicable to multiple hepatic ducts that are severely damaged by tumor invasion or iatrogenic injury or multiple small bile duct openings at the hepatic hilum following extended bile duct resection. ${ }^{13}$ Three technical tips could facilitate an efficient cluster hepaticojejunostomy. The first is to make the multiple bile duct openings wide and parallel after sequential side-to-side unification. The 
second is to radially anchor the traction suture materials at the anterior anastomotic line. The third is to make multiple segmented continuous sutures at the posterior anastomotic line, thus 2 or 3 intervening sutures are usually necessary for most sizable bile duct openings.

In conclusion, hilar PV wedge resection and roofing patch venoplasty is proposed as a useful option to facilitate complete tumor resection in patients undergoing bile duct resection for hepatobiliary malignancy.

\section{CONFLICT OF INTEREST}

None of the authors has any conflict of interest.

\section{ORCID}

Sung-Min Kim: https://orcid.org/0000-0003-2079-6141

Shin Hwang: https://orcid.org/0000-0002-9045-2531

\section{REFERENCES}

1. Chen W, Ke K, Chen YL. Combined portal vein resection in the treatment of hilar cholangiocarcinoma: a systematic review and meta-analysis. Eur J Surg Oncol 2014;40:489-495.

2. Wu XS, Dong P, Gu J, Li ML, Wu WG, Lu JH, et al. Combined portal vein resection for hilar cholangiocarcinoma: a meta-analysis of comparative studies. J Gastrointest Surg 2013;17:11071115.

3. Ebata T, Nagino M, Kamiya J, Uesaka K, Nagasaka T, Nimura Y. Hepatectomy with portal vein resection for hilar cholangiocarcinoma: audit of 52 consecutive cases. Ann Surg 2003;238:
720-727.

4. Sakamoto Y, Yamamoto J, Saiura A, Koga R, Kokudo N, Kosuge T, et al. Reconstruction of hepatic or portal veins by use of newly customized great saphenous vein grafts. Langenbecks Arch Surg 2004;389:110-113.

5. Lin CC, Hsieh CE, Chen YL. Great saphenous vein harvesting for venous outflow reconstruction in living donor liver transplantation - a minimally invasive refinement of the conventional procedure. Int J Surg 2013;11:249-252.

6. Hwang S, Sung KB, Park YH, Jung DH, Lee SG. Portal vein stenting for portal hypertension caused by local recurrence after pancreatoduodenectomy for periampullary cancer. J Gastrointest Surg 2007;11:333-337.

7. Zhou ZQ, Lee JH, Song KB, Hwang JW, Kim SC, Lee YJ, et al. Clinical usefulness of portal venous stent in hepatobiliary pancreatic cancers. ANZ J Surg 2014;84:346-352.

8. Mizuno T, Ebata T, Yokoyama Y, Igami T, Sugawara G, Mori $\mathrm{Y}$, et al. Percutaneous transhepatic portal vein stenting for malignant portal vein stenosis secondary to recurrent perihilar biliary cancer. J Hepatobiliary Pancreat Sci 2015;22:740-745.

9. Hwang S, Ha TY, Jung DH, Park JI, Lee SG. Portal vein interposition using homologous iliac vein graft during extensive resection for hilar bile duct cancer. J Gastrointest Surg 2007;11: 888-892.

10. Hwang S, Ko GY. Right trisectionectomy with en bloc portal vein resection for cholangiocarcinoma after preoperative stenting for main portal vein occlusion. Ann Hepatobiliary Pancreat Surg 2020;24:174-181.

11. Namgoong JM, Hwang S, Oh SH, Kim KM, Park GC, Ahn CS, et al. Living-donor liver transplantation with inferior vena cava replacement in an infant recipient with advanced hepatoblastoma. Ann Hepatobiliary Pancreat Surg 2020;24:72-77.

12. Kwon H, Kwon H, Hong JP, Han Y, Park H, Song GW, et al. Use of cryopreserved cadaveric arterial allograft as a vascular conduit for peripheral arterial graft infection. Ann Surg Treat Res 2015;89:51-54.

13. Hwang S, Ha TY, Song GW, Jung DH. Cluster hepaticojejunostomy with radial spreading anchoring traction technique for secure reconstruction of widely opened hilar bile ducts. Korean J Hepatobiliary Pancreat Surg 2016;20:66-70. 